\title{
Relation between stabilization energy, crystal field coefficient and the magnetic exchange interaction for $\mathrm{Tb}^{3+}$ ion
}

\author{
ZHANG Xiangmu ${ }^{1)}$, MA Weryuan ${ }^{1)}$, CUI Shinven ${ }^{2)}$, and WANG Lihua ${ }^{1)}$ \\ 1) Physics Department, Cangzhou Nomal College, Cangzhou 061001, China \\ 2) Institute of Mechanics, Chinese Academy of Sciences, Beijing 100080, China \\ ( Received 2006-06 22)
}

Abstract: Based on a single ion model, Hamiltonian of the simplest form about magnetocrystalline anisotropy for $\mathrm{Tb}^{3+}$ ion was solved by using the numerical method. The relation between the stabilization energy, crystal field coefficient $B_{2}{ }^{0}$ and the magnetic exchange interaction was studied as temperature approaches to $0 \mathrm{~K}$. The results show that the stabilization energy contributed by $\mathrm{Tb}^{3+}$ is linear with crystal field coefficient $B_{2}{ }^{0}$ approximately, but it is insensitive to the change of magnetic exchange interaction for the strong magnetic substances such as $\mathrm{TbCo}_{5}, \mathrm{~Tb}_{2} \mathrm{Co}_{17}$ and $\mathrm{Tb}_{2} \mathrm{Fe}_{14} \mathrm{~B}$ compounds.

Key words: single ion model; magnet ic exchange interaction; crystal field coefficient; stabilization energy

\section{Introduction}

In the early $1970 \mathrm{~s}$, Greedan and Rao[ 1] had calculated the simplest Hamiltonian based on the magnetocrystalline anisotropy single ion model using the numerical method and present ed the relation between the easy magnetization direction (the easy plane or the easy axis) of rare earth magnetic sublattice and the sign (positive or negative) of crystal field coefficient $B_{2}{ }^{0}$ for $\mathrm{RCO}(\mathrm{R}=$ rare earth $)$ and $\mathrm{R}_{2} \mathrm{Co}_{17}$ cont pounds. On these, the experimental facts of the easy magnetization direction were interpreted for the compounds. Moreover, this method is suit able for other strong magnetization compounds under the allowable condition of ignoring all terms of crystal field coefficient except $\mathrm{B}_{2}{ }^{0} \mathrm{O}_{2}{ }^{0}$. Large numbers of reports show that single ion model explains the magnetocrystalline anisotropy of $4 f$ ion in the rare earth compounds success fully[ $z 4]$.

When the easy and hard magnet ization $\mathrm{d}+$ rection along the axis or the plane, the stabili zation energy of $\mathrm{R}^{3+}$ ion takes the maximum or the minimum of the magnetocrystalline anisotr $\theta$ py energy attributed by the $\mathrm{R}^{3+}$ ion[1]. The main form of the Hamiltonian only contains the crystal field action and magnetic exchange inter action when employing single ion model to cat culate the magnetocrystalline anisotropy energy of 4 ion in rare earth transition metal intermetallic compounds. The crystal field coefficient can be obtained from magnetization measure or calculation, but there is definite deviation. Magnetic exchange interact ion estimated by mot ecule field approximation also exists deviation about 30\% [57]. So it should be a significant research to identify the effect on the magnet $\theta$ crystalline anisotropy by changing crystal field coefficient and magnetic exchange interaction. In this paper, adopting a single ion model, the simplest Hamiltonian of magnetocrystalline anisotropy of $\mathrm{Tb}^{3+}$ ion was calculated by numer ical method. The relation between the stabiliza tion energy, the crystal field coefficient and magnetic exchange interaction was presented under the action of various crystal field and molecule field.

\section{Calculating method}

According to the single ion model, the 
Hamiltonian acting on the ground state wave function of rare earth ion can be written as folt WS:

$H=\sum_{n, m} B_{n}^{m} O_{n}^{m}+2\left(g_{J}-1\right) \mu_{\mathrm{B}} J \cdot H_{\mathrm{ex}}$

The first term of Eq. (1) is the generalized for mulation of crystatfield interaction and the see ond represents the interaction of the rare earth magnetic moment with the exchange field. For the firstgrade approximation, Hamiltonian can be expressed in the simple form:

$H=B_{2}^{0} O_{2}^{0}+G\left(J_{x} \sin \theta+J_{z} \cos \theta\right)$

here,

$G=2\left(g_{J}-1\right) \mu_{\mathrm{B}} H_{\mathrm{ex}}, O_{2}^{0}=3 J_{\mathrm{z}}^{2}-J(J+1)$

Where $B_{2}{ }^{0}$ is crystal field coefficient, $\mathrm{O}_{2}{ }^{0}$ is the Stevens equ ivalent operator [ 89], $H_{\text {ex }}$ repr esents intensity of exchange field acting on the rare earth sublattice. $J, J_{x}, J_{z}$ are total angut lar momentum and its component of $\mathrm{R}^{3+}$ ion re spectively, and $\theta$ is the angle between $H_{\text {ex }}$ and the $c$ axis.

For $\mathrm{Tb}^{3+}$ ion, $J=6$, select values of $B_{2}{ }^{0}$ and $G$, then act Eq. (2) on the grountstate wave function of $\mathrm{Tb}^{3+}$ ion and adopt numerical method, thirteen splitting values $E_{i}(\theta) \quad(i=$ $1,2,3, \ldots \ldots, 13)$ can be obtained. A ccording to the free energy expression given by Helmheltz, the magnetocrystalline anisotropy energy of $\mathrm{Tb}^{3+}$ ion is:

$E_{\text {an }}(\theta, T)=-k T \ln \sum \exp \left[-E_{i}(\theta) / k T\right]($

Stabilization energy:

$E_{\text {st }}(T)=E_{\text {an }}\left(90^{\circ}, T\right)-E_{\text {an }}\left(0^{\circ}, T\right)$

As $T \rightarrow 0 \mathrm{~K}$,

$E_{\mathrm{st}}(0)=E_{\text {an }}\left(90^{\circ}\right)-E_{\text {an }}\left(0^{\circ}\right)$

\section{Results and discussion}

From Eqs. (2) - (5), the numerical rela tion between $E_{\text {st }}$ and $B_{2}{ }^{0}$ can be obtained by given values of $\mathrm{G}$ arbitrarily as $T \rightarrow 0 \mathrm{~K}$. Simi larly, relation between stabilization energy and magnetic exchange interaction can be obtained by given values of $B_{2}{ }^{0}$ arbitrarily. For advart tage of analysis, some results are plotted in Figs. 1 and 2.

Just as shown Figs. 1 and 2, for different G, $E_{\text {st }}>0$ as $B_{2}{ }^{0}<0$, which implies the easy magnetization direction of $\mathrm{Tb}$ lattice is parallel to $e$ axis; but $E_{\text {st }}<0$ as $B_{2}{ }^{0}>0$, which ex presses the easy magnetization direction is per pendicular to $c$-axis. The results show quite good agreement with those obtained by Greedan and Rao[1] .

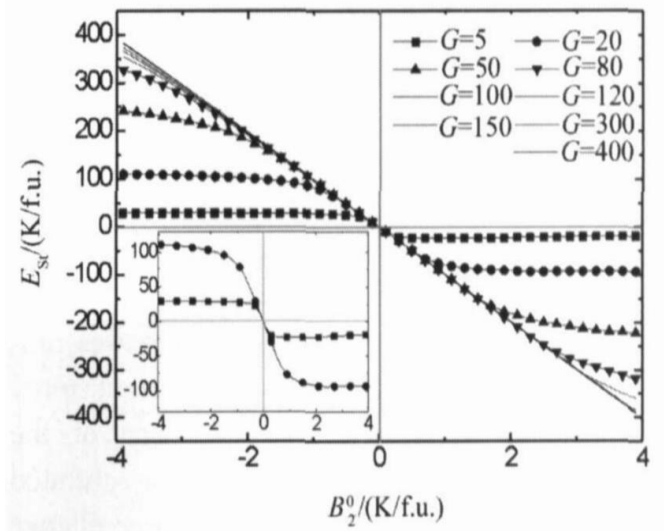

Fig. 1. Relation between stabilization energy and crystal field coefficient for given $G(\mathrm{~K} / \mathrm{f} . \mathrm{u}$.$) ) (1 \mathrm{~K}$ can be converted to $\left.1.381 \times 10^{-23} \mathrm{~J}\right)$.

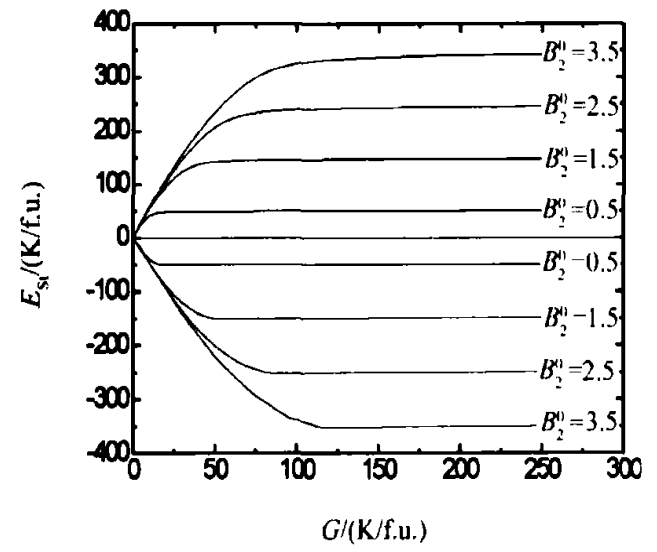

Fig 2. Relation between stabilization energy and magnetic exchange interaction for given $B_{2}{ }^{0}(K /$ f. u. $)$.

Fig. 1 displays the variations of stabiliza tion energy with crystal field coefficient for $G=$ $5, \quad 20,50,80,100,120,150,300$, $400 \mathrm{~K} / \mathrm{f}$. u. . From Fig. 1, the variety of stabili zation energy of $\mathrm{Tb}^{3+}$ ion with crystal field coef ficient $\mathrm{B}_{2}{ }^{0}$ is very complex and does not exhibit any clear simple functional relations when vat ues of $G$ are lower. The smaller graph embect ded in Fig. 1 shows details of variation stabiliza tion energy with crystal field coefficient for $G=$

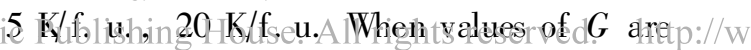


higher ( such as $G>80 \mathrm{~K} / \mathrm{f}$. u. ), stabilization energy of $\mathrm{Tb}^{3+}$ ion is linear with crystal field coefficient $\mathrm{B}_{2}{ }^{0}$ approximately, showing as the curves when $G=100,120,150,300$, $400 \mathrm{~K} / \mathrm{f}$. u. in Fig. 1. More calculations indicate $E_{\text {st }} / B_{2}{ }^{0} \approx$ constant as $G>80 \mathrm{~K} / \mathrm{f}$. u. .

Fig. 2 displays the changing curves of sta bilization energy with magnetic exchange inter action when $B_{2}{ }^{0}= \pm 0.5, \pm 1.5, \pm 2.5$, $\pm 3.5 \mathrm{~K} / \mathrm{f}$. u. For any curve in Fig. 2, the whole changing range for values of $G$ can be $d \dot{t}$ vided sketchily into three regions according to the variety of stabilization energy: (a) no-satt rated region, stabilization energy increases rapidly with increasing $G$ from the start of zero, with further increasing $G$, the slope of the curve decreases gradually; (b) quasi saturated region, more bigger of $G$, more slower change of stabilization energy; (c) saturated region, when values of $G$ increase further, stabilization energy do not exist obvious changing pattern. If the $G$ at which the stabilization energy reaches $95 \%$ of its maximum is defined as the left boundary of quasi saturated region, then the dependence of stabilization energy on $G$ is weak in the quast saturated region and saturated re gion for any curve. In addition, it can be seen from Fig. 2, smaller the $\left|B_{2}{ }^{0}\right|$ is, smaller the corresponding ne saturated region is .

For strong magnetic substance, TbCos $\left(B_{2}{ }^{0}=1.4 \mathrm{~K} /\right.$ f. u. , $G=210 \mathrm{~K} /$ f. u. $)[1]$, $\operatorname{Tb}_{2} \operatorname{Co}{ }_{17}\left(B_{2}{ }^{0}=0.43 \mathrm{~K} / \mathrm{f}\right.$. u.,$\quad G=210 \mathrm{~K} / \mathrm{f}$. u. $)[1], \mathrm{Tb}_{2} \mathrm{Fe}_{14} \mathrm{~B}\left(\mathrm{~B}_{2}{ }^{0}=-2.4 \mathrm{~K} /\right.$ f.u., $G$ $=152 \mathrm{~K} /$ f.u. ) [10], values of $G$ of $\mathrm{Tb}^{3+}$ ion are in quasi-saturated region or saturated re gion, so the variation of stabilization energy of $\mathrm{Tb}^{3+}$ ion is linear with crystal field coefficient $\mathrm{B}_{2}{ }^{0}$ approximately, but the variation of magnet ic exchange interaction has no obvious effect on the stabilization energy.

\section{Conclusions}

As temperature approaches to $0 \mathrm{~K}$, for weak magnetic substances, the variety of stabi lizat ion energy of $\mathrm{Tb}^{3+}$ ion with crystal field $\mathrm{co}$ efficient $\mathrm{B}_{2}{ }^{0}$ and magnet ic exchange interaction is more complex. But for strong magnet ic substances, variation of stabilization energy of $\mathrm{Tb}^{3+}$ ion is linear with crystal field coefficient $\mathrm{B}_{2}{ }^{0}$ and insensitive to the change of magnet ic exchange interaction.

Adknowledge: We thank professor ZHANG Zhongwu for providing partial supports.

\section{Ref erences}

[1] Greedan J. E., and Rao V. U.S., An analysis of the rare earth contribution to the magnetic anisotropy in $\mathrm{RCo}_{5}$ and $\mathrm{R}_{2} \mathrm{Co}_{17}$ compounds. Journal $g$ Solid State Chemistry, 1973, 6: 387.

[2] Franse J. J. M. , and Radwanski R. J. , Magnetic properties of binary rare earth $3 \mathrm{~d}$ transitionmetal intemetallic compounds, [ in] Buschow K H J. Handbook of Magnetic Materials (Vol. 7), NorthHolland, Amsterdam, 1993: 307.

[3] Zhang Zhongwu, Dang Meizhen, and Li Huaishan, Analysis of the spin reorientation in $\mathrm{R}_{2} \mathrm{Fe}_{14} \mathrm{~B}$ and $\mathrm{Tb}_{2}\left(\mathrm{Fe}_{0.9} \mathrm{M}_{0.1}\right)_{14}$ B. J. Magn. Magn. Mater., 1989, 81: 361.

[4] Zhang Zhongwu, Huang Ruiwang, and Dang Meizhen, The magnetic anisotropy of $R_{2} \mathrm{Fe}_{14} \mathrm{~B}$ $(\mathrm{R}=\mathrm{Tb}, \mathrm{Pr})$ and error analysis. J. Magn. Magn. Mater., 1990, 92: 196.

[5] Kirchmayr H. R., and Poldy C. A., Magnetism in rare earth 3d intemetallics. J. Magn. Magn. Mater., 1978, 8: 1.

[6] Ibarra M. R., Algarabel P.A., Marquina C., et al., Magnetic phase diagram and anisotropy of pseudoternary ( $\left.\mathrm{Er}_{x} \mathrm{Dy}_{1-x}\right)_{2} \mathrm{Fe}_{14} \mathrm{~B}$ compounds. Phys. Rev. B, 1989, 39: 781.

[7] Wang J. L., GarciaLanda B., Marquina C., et $a l .$, Spin reorientation and crystatfield interaction in $\mathrm{TbFe}_{12-} \mathrm{Ti}_{x}$ single cystals. Phys. Re . B, 2003, 67: 014417 .

[8] Stevens K. W. H., Matrix elements and operator equivalents connected with the magnetic properties of rare earth ions. Proc. Phys. Soc. (London), 1952, A65: 209.

[9] Smith D., and Thornley J. H. M. , The use of ' operator equivalents'. Proc. Phys. Soc., 1966, 89: 779.

[10] Radwanski R. J. , and Franse J. J. M., Rare earth contribution to the magnetocryst alline anisot ropy en ergy in $\mathrm{R}_{2} \mathrm{Fe}_{14}$ B. Phys. Rev. B, 1987, 36: 8616 . 